\title{
The Research and Application of City Emergency System for Medium and Small Cities
}

\author{
Yu LI ${ }^{1, a}$, Chun CHEN ${ }^{1, b,{ }^{*}}$, Dong ZHONG ${ }^{2, c}$ and Ruolin RUAN ${ }^{3, d}$ \\ ${ }^{1}$ College of physical education, Hubei University of Science and Technology, Xianning, China; \\ ${ }^{2}$ College of electronic and information, Hubei University of Science and Technology, Xianning, China; \\ ${ }^{3}$ College of biomedical engineering, Hubei University of Science and Technology, Xianning, China;
}
a27820424@163.com, ㅁ006572183@qq.com, ' zhongdong0129@163.com, d251824144@qq.com
${ }^{*}$ Corresponding author

Keywords: Medium and small cities, Emergency system, System architecture

\begin{abstract}
Medium and small cities are the high proportion in the total number of cities in China, the con-struction of emergency system for medium and small cities is relatively backward compared with big cities, speeding up the construction of medium and small cities emergency system is imperative. This paper proposes a new design scheme based on the analysis of the traditional medium and small cities emergency system construction, which is more suitable for medium and small cities. The system architecture, the hierarchical structure and business process have been designed in this paper. It provides a good research direction for the construction of emergency system for medium and small cities.
\end{abstract}

\section{Introduction}

In recent years, with the rapid development of the social and economy, the city public security becomes the focus of attention, Domestic city emergencies such as earthquakes, production safety accidents, malignant diseases spreading, natural disasters and other unexpected events occur frequently ${ }^{[1]}$, which affected seriously city public security. In order to reduce the losses of emergency events, the construction of emergency response system is imperative. At present domestic some big cities, for example, Nanning, Beijing, Tianjin, Guangzhou, Shenzhen, Shanghai and other large and super large cities have been completed the emergency response system, which are responsible for public emergencies $^{[2]}$. Due to smaller scale, the lack of financial resources, construction mode and system construction for medium and small cities are relatively backward. Except for the public security and fire department, the information systems of medium and small cities emergency departments are backward. It is necessary to speed up the construction of primary emergency system and to provide better service to the people ${ }^{[3]}$. This paper proposes a new design scheme based on the analysis of the traditional medium and small cities emergency system construction, which is more suitable for medium and small cities. The system architecture, the hierarchical structure and business process have been designed in this paper. It can provide a good research direction for the construction of emergency system for medium and small cities.

\section{The system architecture design of medium and small city emergency system}

In general, the emergency system of big cities is composed of wireless communication system, wireless communication voice dispatching system, integrated response alarm system, voice recording 
system, wireless data transmission system, video image system, city geographical information system, moving target positioning system, mobile communication system ${ }^{[4]}$. The emergency system has the command dispatch function. It can achieve the rapid reaction and unified response alarm to natural disasters, accidents disasters, public health emergencies and emergency public social security. It also can provide communications and information security for the decision of super-serious events ${ }^{[5][6]}$. But the traditional emergency systems of medium and small cities cannot achieve the integration of resources, lack of unified management and lack of unified scheduling. The efficiency of response alarm is low.

This paper proposes the new system architecture, which is suitable for medium and small city emergency system according to the characteristics of medium and small cities in China. Figure 1 shows the system architecture diagram designing for medium and small cities emergency system, the system architecture has been divided roughly into three parts: command application layer, linkage department data layer and infrastructure layer.

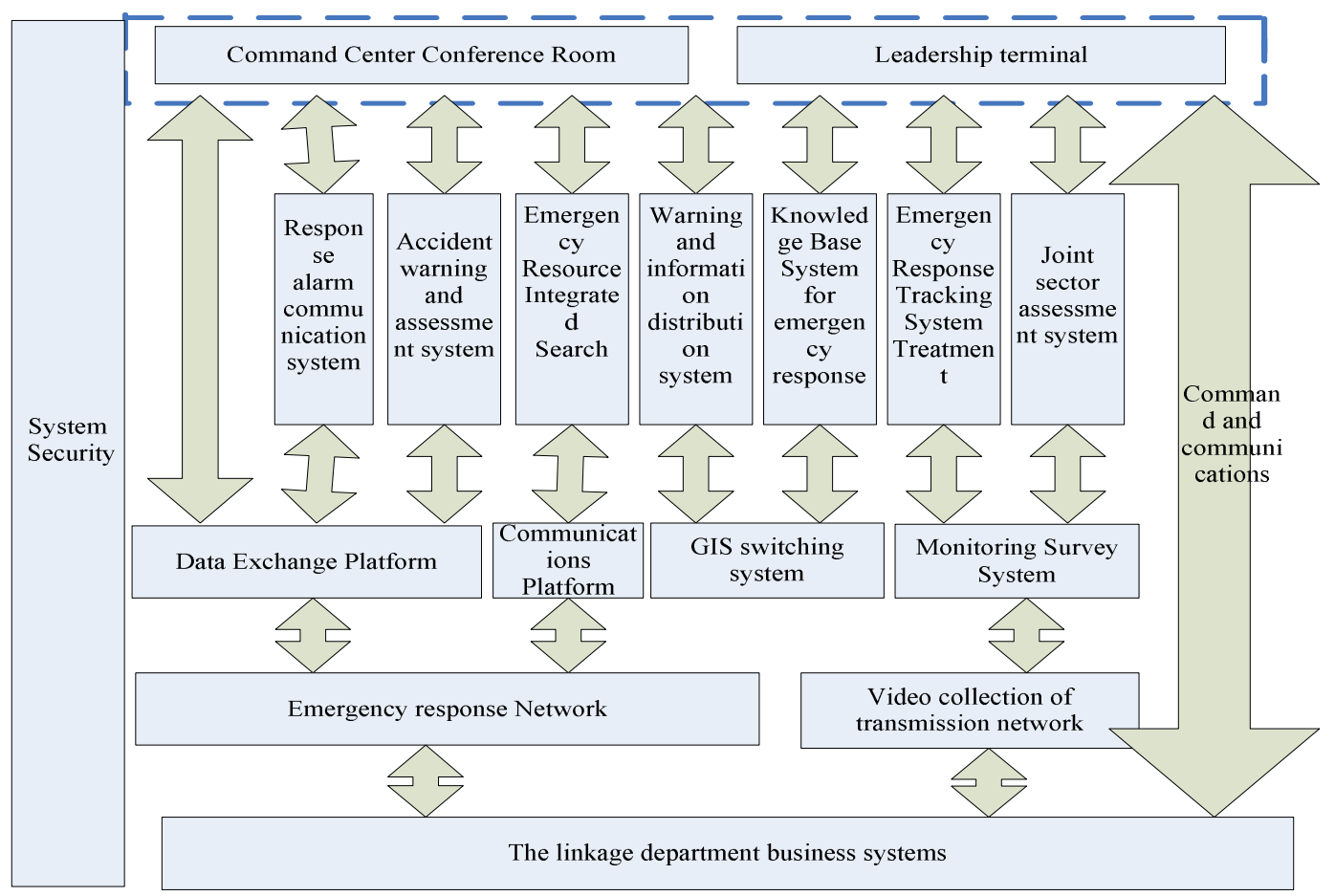

Figure1. Small urban emergency system architecture diagram

In the paper, the center of emergency system architecture is composed mainly of response alarm system, emergency resources comprehensive search system, accident warning and evaluation system, warning and information release system, the emergency treatment knowledge base system, emergency response processing tracking system, the linkage department evaluation system, the functions of main systems are as follows:

1) The response alarm communication system has the phone scheduling, computer network scheduling function and wireless scheduling function. It is prior to use network scheduling in normal circumstances of public security network, and if the network is impassability, the system would be inferred promptly to use traditional telephone scheduling automatically. It integrated with all communication systems to constitute a complex system, which achieved convenient and interoperable voice communication function. The response alarm communication system is the wireless scheduling system of all communication terminal, which including response alarm call center, digital simulation 
cluster communication equipment, cell phone, public telephone, and so on.

2) Emergency resources comprehensive search system, which can search quickly the storage device data and provide services. It can search specific surveillance video and associated data of government in the Web interface by the ways of lens, time, association events, log and so on. It can provide unified management for the emergency resources of the governments and the city leaders can use the system to make decision quickly according to on-site conditions and provide handling for emergency event by mobilizing related resources through the shortest path.

3) Accident warning and evaluation system can provide regulations and system inquires according to the type of emergencies, and it can define the early warning index and assessment criteria of emergencies. At the same time, the system can make the standard of the early warning index and disaster grade evaluation of emergency.

4) Some information can be posted on the WEB by the alarm and information release system and the related departments can share the information. The system can transmit the information to all kinds of medium in the first time, which can not only release information to the public, but also can get the public provided clues.

5) Emergency treatment knowledge base system, the operation data of this system is sensitive and classified information. In the system the professional data must be stored by mutual isolation distribution and important data must be encryption and even physical isolation. It is to complete the collecting, classification and comprehensive utilization of emergency response knowledge.

6) Emergency response processing tracking system, various linkage department personnel and onsite command staff must provide the latest information of dealing with emergencies to government command center report meeting room through the network, and it can also using the mean of the fixed telephone and mobile phone. It could be accessible to get the information of the working condition, onsite condition, instruction state for all departments and disposal team, even personal through the tracking systems.

7) The linkage department evaluation system, the self-commitment and management is stressed in this system. The operation is very simple and it is more suitable for the departments of not quantitative unit. The system can complete the evaluation emergency response work of all departments and promote the construction of emergency response command system for the linkage department, improving the handling level of emergency event.

\section{The hierarchical structure design of medium and small city emergency system}

The paper presents the hierarchical structure design of medium and small city emergency system: the core layer, institutions layer, communication layer, directors, relevant layer. All levels have different data and applications. Its hierarchical structure has been shown in Figure2: 


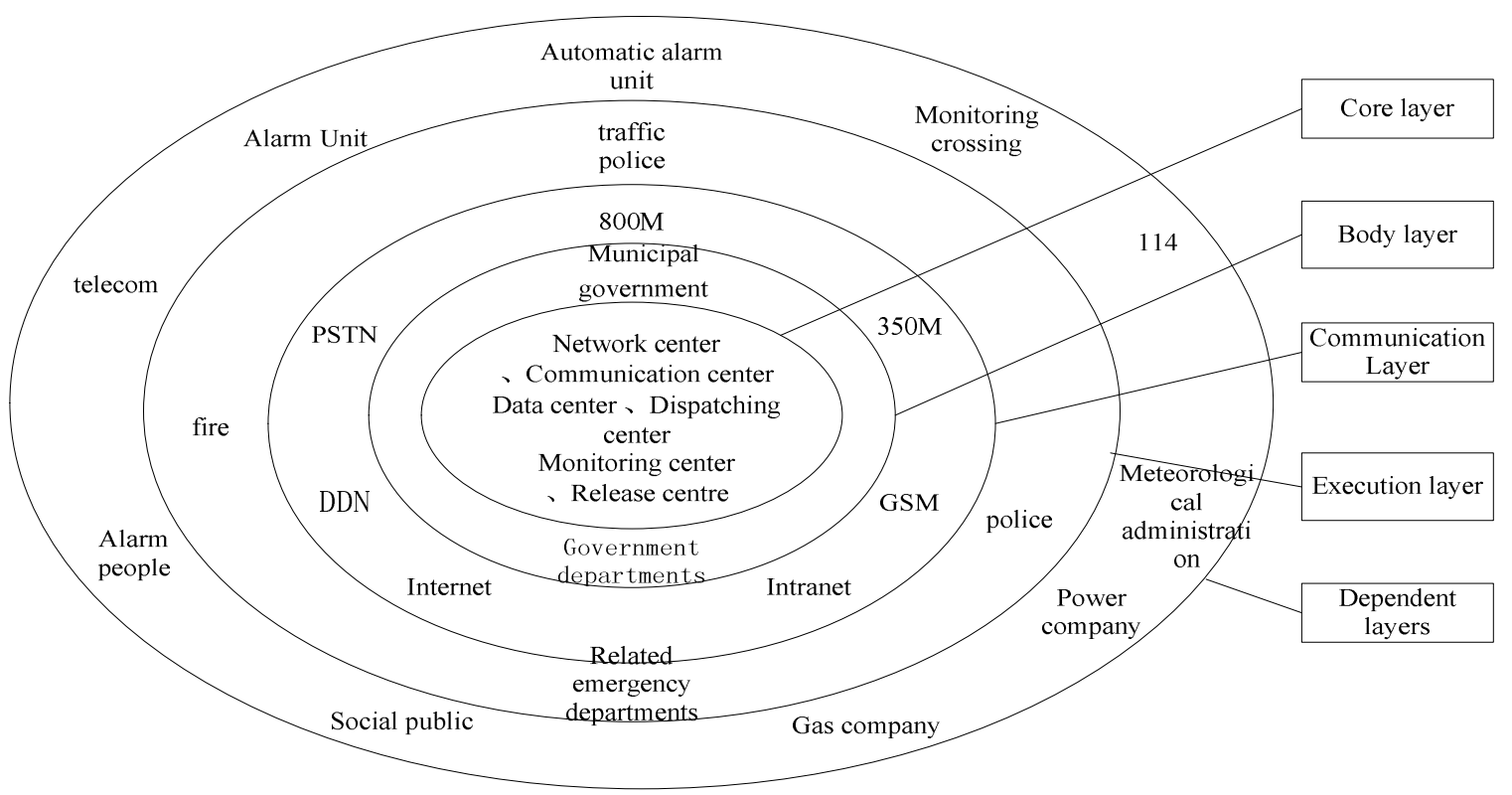

Figure 2 the emergency system structure diagram of small and medium city

1) The core layer is the command center of the emergency system. It is composed of dispatching center, network center, communications center, the monitoring center, data center and release center. Command center can achieve the integrated application of database, comprehensive application of various department data, real-time monitoring of data and image display, application of electronic map display and so on.

2) Institutions layer contains leadership and authority agencies. In the information construction, as a leading unit, it should arrange the communication system, computer network, GPS system, image monitoring system and information resources overall citywide.

3) Communications layer contains all sorts of cable and wireless communications, Digital Data Network (DDN Data), Public Switched Telephone Network (PSTN), wireless trunking communications and so on.

4) Director layer is an information receiver and scheduling command performer in the function. The emergency team is a network sub-center node to distributed database and distributed network in the technology. The emergency team is a data update operator in the management.

5) Related layer refers the individuals or department which is related with the alarm people, alarm unit, meteorology, telecom departments, electric power sector and gas sector and other related with emergency.

\section{The business process design of medium and small city emergency system}

A typical emergency response business process contains six links parts, including call the police, response the police, response alarm, disposal, sources of withdrawal and summarizes. The process has been shown in Fig.3: 


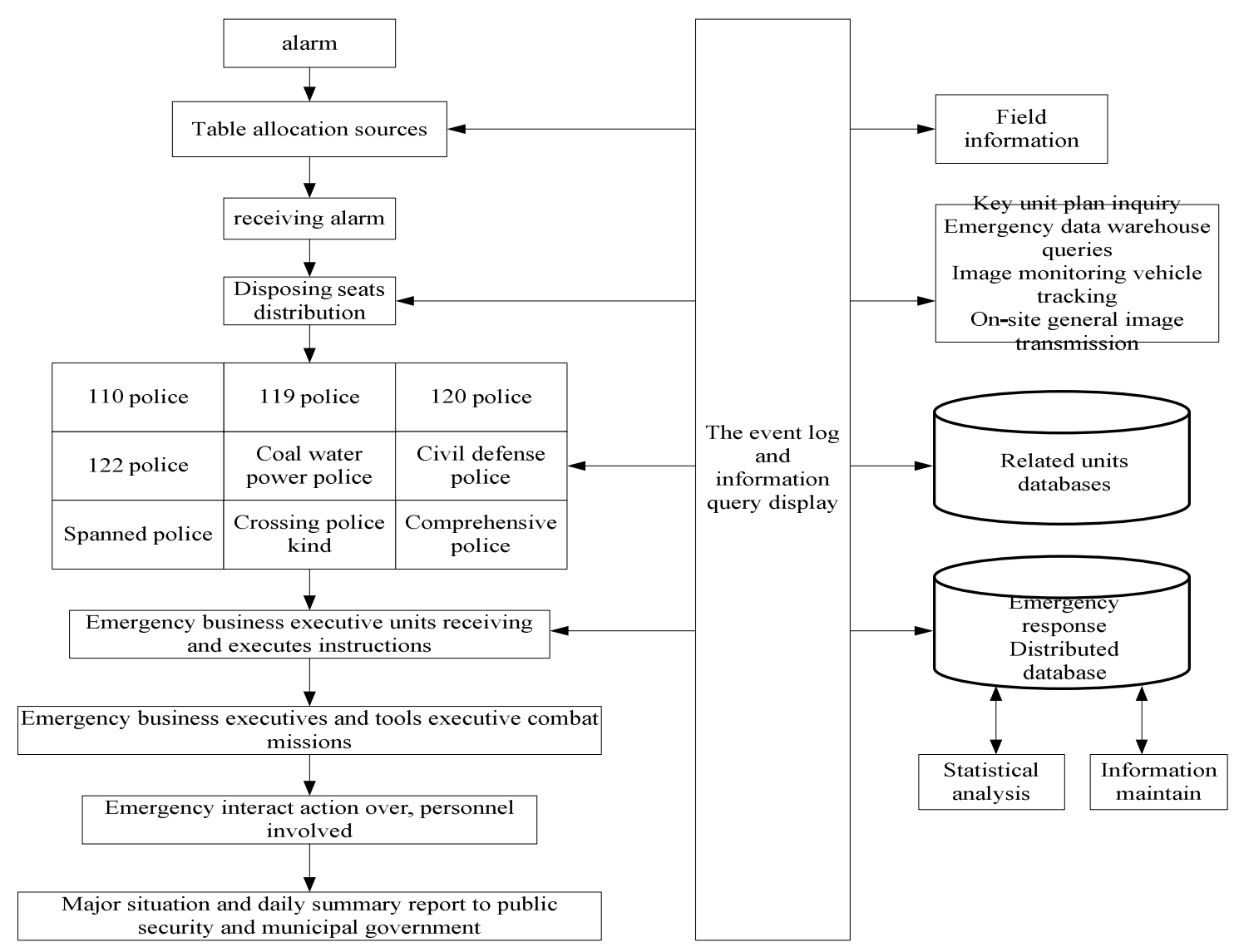

Figure 3 city emergency system business process

when the command center has an acknowledge of happening nonemergency event, it will turn to the convenient service center directly and distributing processing. When the convenient service center received emergency for help, it will also turn to the command center directly. In the disposal of emergency / non emergency, the command center and convenient service center can share information. The decision-making command center is usually one on duty, when encountering major emergency or nonemergency events need coordinate, the attendant report the information to the director of decisionmaking command center, the director will judge the events and report to the city leaders, then the leader will has a decision. When leaders have arrived to command center for decision-making, the system will provide information collection and leading comprehensive coordination communication platform.

\section{Summary}

This paper proposes a new design scheme based on the analysis of the traditional construction for medium and small cities emergency system, which is more suitable for medium and small cities. The system architecture, the hierarchical structure and business process have been designed in this paper. It provides a good research direction for the construction of emergency system for medium and small cities. This system has been applied in Jianli, WuXue medium and small cities, and so on. The system is stability and security. 


\section{ACKNOWLEDGMENTS}

The authors gratefully acknowledge the financial support for this work provided by the colleges and universities of Hubei Province in 2014 the Provincial College Students' innovation and entrepreneurship training plan No. 201410927029 and 201410927025, the National Natural Science Foundation of China (NSFC) under the Grant No. 61271256, the Team Plans Program of the Outstanding Young Science and Technology Innovation of Colleges and Universities in Hubei Province, Grant No.T201513, the Program of the Natural Science Foundation of Hubei Province, Grant No. 2015CFB452, the Scientific research project in Hubei province department of education, Grant No. B2015077.

\section{References}

[1] Zhao Qi, Liu LiQun, Zhao GenMing, etc. Our country emergent public health event emergency situation of early warning system [J]. schisto somiasis prevention magazine, 2006, 18(3): 211-213.

[2] ZhongDong, Chen chun. The study of scheduling function optimization for trunking communication system in CERS [J]. Science technology and engineering, 2011, 18(3): 211-213

[3] Deng Shoupeng. City emergency system management and development [J]. Digital communication world, 2007 (4): 18-20.

[4] Jiang Jianhua Yi Xianhuai, Liu Aiguo. Public health system present situation and countermeasure thinking [J]. Journal of Chinese dietetic hygiene and health, mar 2005 (4): 1-4.

[5] Wang Yue. Construction of the complex information for multiple views - active agents [J]. China Science, 2006, 8 (5): 30-34.

[6] Wei Jun. Security incident michmash, emergency treatment system analysis [J]. Computer engineering, 2004.(7) 